\title{
Prevalence of self-medication among academics of the healthcare field in a university in the middlewest of Santa Catarina before and after the pharmacology discipline
}

\section{Prevalência de auto-medicação entre académicos do campo da saúde numa universidade do centro-oeste de Santa Catarina antes e depois da disciplina de farmacologia}

\section{Karla Maffessoni}

Acadêmica de Medicina pela Universidade Alto Vale do Rio do Peixe (UNIARP) Instituição: Universidade Alto Vale do Rio do Peixe (UNIARP)

Endereço: Rua Victor Baptista Adami, 800 - Centro, Caçador - SC, Brasil

E-mail: karlamaffessoni@hotmail.com

\section{Fábio Herget Pitanga}

Mestre em Desenvolvimento e Sociedade pela Universidade Alto Vale do Rio do Peixe (UNIARP) Instituição: Universidade Alto Vale do Rio do Peixe (UNIARP) Endereço: Rua Victor Baptista Adami, 800 - Centro, Caçador - SC, Brasil E-mail: clinimedsc@yahoo.com.br

\section{ABSTRACT}

Self-medication is characterized by self-administration of drugs that have not been prescribed by the doctor, thereby seeking rapid relief of symptoms. This irrational and incorrect practice can lead to several consequences, such as masking evolutionary diseases, causing dependence, intoxication and drug interactions. The incidence of self-medication is problematized with health students, since they should be aware of such a practice, the risks and harms it may entail. This study aimed to analyze the prevalence of self-medication in nursing, pharmacy and medicine students in Alto Vale do Rio do Peixe University (UNIARP) and what influence the discipline of pharmacology has on the selfadministration of drugs. The results of this analysis may motivate actions in order to bring improvements regarding the correct use of medication among university students and avoid self-medication.

Keywords: Self-medication, Health students, Self administration.

\section{RESUMO}

A automedicação caracteriza-se pela autoadministração de medicamentos que não tenham sido prescritos pelo médico, buscando com isso o alivio rápido de sintomas. Essa prática irracional e incorreta pode acarretar em diversas consequências como, por exemplo, mascarar doenças evolutivas, causar dependência, intoxicações e interações medicamentosas. Problematiza-se a incidência de automedicação com estudantes da área da saúde, uma vez que os mesmos deveriam estar cientes de tal prática, os riscos e malefícios que ela 
pode acarretar. Esse estudo teve como objetivo analisar a prevalência da automedicação em acadêmicos dos cursos de enfermagem, farmácia e medicina na Universidade Alto Vale do Rio do Peixe (UNIARP) e qual a influência que a disciplina de farmacologia exerce sobre a autoadministração dos fármacos. Os resultados dessa análise poderão motivar ações no intuito de trazer melhorias quanto ao uso correto de medicamentos entre estudantes universitários e evitar a automedicação.

Palavras-chave: Automedicação, Acadêmicos da saúde, Autoadministração.

\section{INTRODUÇÃO}

A automedicação tem como princípio a ingestão de fármacos sem acompanhamento ou informações prévias de um profissional qualificado, onde o próprio indivíduo seleciona um medicamento que considera adequado para melhorar seus sinais e sintomas. Esta é uma prática que está presente em diversas culturas e faixas etárias sendo uma das principais causas de intoxicação medicamentosa, efeitos colaterais negativos, havendo também o risco de mascarar doenças evolutivas, já que o fármaco pode esconder manifestações de algumas patologias (GAMA; SECOLI, 2017).

Mesmo que a automedicação faça parte do autocuidado, é um problema de grande proporção que não está restrito apenas aos leigos, pois profissionais da área da saúde fazem uso dessa prática por terem maior acesso e informações sobre os medicamentos (SANTOS et al., 2012). A farmacologia, ciência que estuda o efeito das substâncias químicas no organismo, é uma matéria que está inclusa na grade curricular dos cursos de enfermagem, farmácia e medicina, pois são profissões que lidam direta e indiretamente com a prescrição de fármacos (SANTOS et al., 2015).

Embasado nessa premissa, a presente pesquisa propõe identificar a prevalência da automedicação entre os acadêmicos da área da saúde da Universidade do Alto Vale do Rio do Peixe antes e após a disciplina de farmacologia, quais as classes medicamentosas mais utilizadas e se houve interrupção do uso após aprender mais sobre os fármacos. 


\section{METODOLOGIA}

Trata-se de um estudo de corte transversal, quantitativo. Esse projeto foi aplicado a acadêmicos dos cursos de enfermagem, farmácia e medicina da Universidade do Alto Vale do Rio do Peixe, na cidade de Caçador/SC, através de um levantamento de dados utilizando um questionário estruturado, padronizado e validado no artigo "Perfil da automedicação no Brasil" publicado na Revista de Saúde Pública e utilizado em outros estudos como no "Perfil da automedicação nos pacientes otorrinolaringológico", divulgado na Revista Brasileira de Otorrinolaringologia e no "Estudo comparativo do uso da automedicação entre universitários da área da saúde e universitários de outras áreas não relacionados à saúde na universidade de Marília-SP”, publicado no Brazilian Journal of Development. O questionário utilizado foi adaptado para o desenvolvimento deste estudo.

A população de acadêmicos matriculados antes da disciplina de farmacologia nos cursos de farmácia, enfermagem e medicina é de 133 indivíduos e após a disciplina de farmacologia é de 98. Desta forma, o cálculo amostral da população em estudo para antes da disciplina de farmacologia é de 99 indivíduos e para após a disciplina é de 79. Para o cálculo foi utilizado um grau de confiança de $95 \%$ e $5 \%$ de margem de erro.

Desta forma, foram enviados o questionário e o termo de consentimento informado para todos os alunos elegíveis, por via online. A coleta de dados foi realizada no segundo semestre de 2021, para os alunos do primeiro e décimo período dos cursos de farmácia e enfermagem, assim como para os alunos do primeiro e sexto período do curso de medicina, tendo em vista que por ser um curso novo não possui turmas à frente. Afim de evitar vieses, definiu-se como critérios de exclusão alunos reprovados ou que estejam impossibilitados de cursar a disciplina de farmacologia, assim como alunos cujos questionários tenham sido preenchidos de forma incompleta, errada ou com alguma inconsistência.

Para os cálculos estatísticos utilizou-se o programa Statistical Package for the Social Science (SPSS), versão 22.0 for Windows. 


\section{RESULTADOS E DISCUSSÕES}

Foram entrevistados 71 acadêmicos dos cursos de medicina, enfermagem e farmácia da Universidade do Alto Vale do Rio do Peixe, do primeiro, sexto e décimo períodos. Dessa forma, totalizando 39 alunos que já cursaram a disciplina de farmacologia e 32 que ainda não cursaram. A frequência de automedicação entre os participantes da pesquisa é de $91,5 \%$, conforme apresentada na Tabela 1 , sendo que $54,9 \%$ compravam os fármacos baseandose em receitas médicas antigas e $90,1 \%$ relatam que não houve alguma implicação negativa com o uso dessas medicações.

Tabela 1 - Número e porcentagem de acadêmicos divididos por períodos que alegam ou não fazer uso da automedicação e para quem era o uso.

\begin{tabular}{lcc} 
& \multicolumn{2}{c}{$\begin{array}{c}\text { Número de Entrevistados e } \\
\text { Porcentagem por Periodo } \\
\mathbf{1}^{\circ} \text { periodo }\end{array}$} \\
\hline Para uso próprio & $21(65,6 \%)$ & $20(51,2 \%)$ \\
\hline $\begin{array}{l}\text { Uso próprio e para } \\
\text { membros da familia }\end{array}$ & $5(15,6 \%)$ & $19(48,8 \%)$ \\
\hline
\end{tabular}

Não fazem uso

Uma porcentagem expressiva de alunos interrogados no presente estudo informou já ter indicado medicações para familiares. Percebeu-se ainda que dos alunos que ainda não cursaram a disciplina de farmacologia ( $1^{\circ}$ período) $15,6 \%$ já fizeram indicação de fármacos e para os alunos que já cursaram a disciplina ( $6^{\circ}$ e $10^{\circ}$ períodos) os dados coletados foram de $48,8 \%$. Um estudo conduzido na Arabian Gulf University mostrou que os estudantes de medicina que se encontram em etapas mais avançadas do curso também possuem essa prática de forma mais exacerbada (JAMES et al., 2008)

Os medicamentos mais utilizados para automedicação são analgésicos e antitérmicos, anti-inflamatórios e medicamentos para resfriado/gripes, conforme descrito no Gráfico 1. Notam-se dados semelhantes a Galato et al. (2012) onde 
o estudo refere que analgésicos (28\%) e antigripais (16,0\%) são os remédios mais solicitados sem receita.

\section{Gráfico 1 - Proporção de quais medicamentos foram comprados para automedicação}

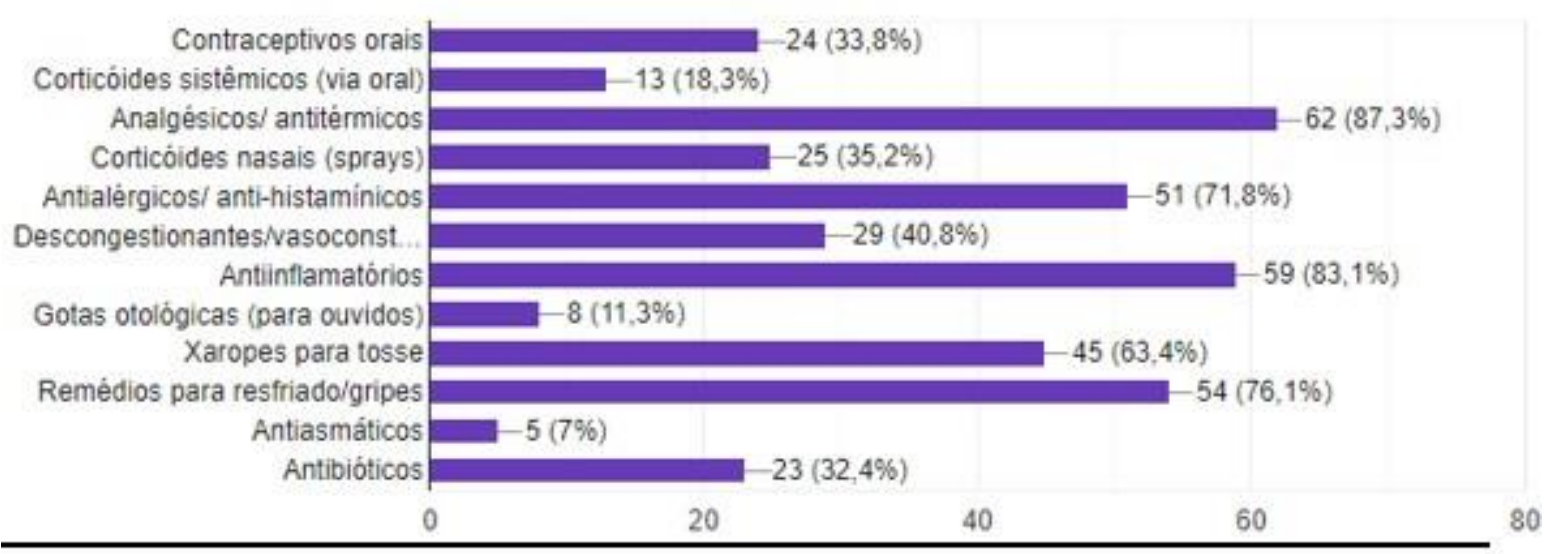

Fonte: Os autores (2021)

Ainda de acordo com os autores (GALATO et al., 2012), farmacêuticos ou funcionários da farmácia tem grande influência na automedicação pois são responsáveis pela maioria das indicações medicamentosas. Esse dado foi evidenciado na presente pesquisa quando a população estudada relatou que $78,9 \%$ já aconselharam-se com o farmacêutico ou balconista para comprar medicações e $77,5 \%$ receberam conselhos não solicitados.

\section{CONSIDERAÇÕES FINAIS}

O presente estudo teve como objetivo analisar a prevalência da automedicação em acadêmicos dos cursos de enfermagem, farmácia e medicina na Universidade do Alto Vale do Rio do Peixe e qual a influência que a disciplina de farmacologia exerce sobre a autoadministração dos fármacos. Apesar da baixa aderência, observou-se que é consideravelmente alto o número de estudantes que fazem uso de medicação sem prescrição médica e que além do uso próprio, os alunos que já cursaram a disciplina também indicam com maior incidência medicações para familiares. Os resultados desses estudo podem ser utilizados para motivar ações no intuito de trazer melhorias quanto ao uso correto 
de medicamentos entre estudantes universitários a fim de evitar a automedicação.

\section{INSTITUIÇÕES FINANCIADORAS E APOIADORAS / AGRADECIMENTOS}

Agradeço ao Fundo de Apoio a Pesquisa da Universidade do Alto Vale do Rio do Peixe - FAP/UNIARP, Secretaria de Saúde pela concessão de Bolsas de Iniciação Cientifica, aos cursos de Enfermagem, Medicina e Farmácia da UNIARP pela disponibilização dos dados necessários. 


\section{REFERÊNCIAS}

Galato, dayani; madalena, jaqueline; pereira, greicy borges. Automedicação em estudantes universitários: a influência da área de formação. Ciênc. Saúde coletiva, rio de janeiro, v. 17, n. 12, p. 3323-3330, dez. 2012.

James $\mathrm{h}$. Et al. Influence of medical training on self-medication by students. Int journ of clinic pharm and therap, vol. 46,1, p. 23-29, 2008.

Gama, abel santiago muri; secoli, silvia regina. Automedicação em estudantes de enfermagem do estado do amazonas - brasil. Rev. Gaúcha enferm., porto alegre, v. 38, n. 1, e65111, 2017.

Santos, b. Et al. Incidência da automedicação em graduandos de enfermagem. Journal of the health sciences institute, v. 30, n. 2, p. 156-160, 2012.

Santos, maria aparecida et al. Entendendo a farmacologia: conceitos básicos. Unilus ensino e pesquisa, v. 12, n. 28, p. 119, 2015.

Servidoni, a. B. Et al. Self-medication profile of ent patients. Brazilian journal of otorhinolaryngology, v. 72, n. 1, p. 83-88, jan. 2006.

Tarley, m. G. G et al. Estudo comparativo do uso da automedicação entre universitários da área da saúde e universitários de outras áreas não relacionados à saúde na universidade de marília-sp. Brazilian journal of development, vol.23, n.1, pp.22-27, 2018. 